\title{
Sex differences in the relationships between body dissatisfaction, quality of life and psychological distress
}

\author{
Scott Griffiths, ${ }^{1,2}$ Phillipa Hay, ${ }^{3}$ Deborah Mitchison, ${ }^{3,4}$ Jonathan M. Mond ${ }^{3,4}$ Siân A. McLean, ${ }^{5}$ Bryan Rodgers, ${ }^{6}$ \\ Robin Massey, ${ }^{5}$ Susan J. Paxton ${ }^{5}$
}

ody dissatisfaction, referring to subjective dissatisfaction with body size and/or shape, has recently been recognised as a public health problem. ${ }^{1,2}$ Previously, most studies of body dissatisfaction focused on its role as a risk factor for the development of other adverse psychological phenomena, including eating disorders ${ }^{3}$ and depressive symptoms. ${ }^{4}$ Population level studies of body dissatisfaction have largely been confined to adolescent girls and young women, ${ }^{3}$ despite evidence that constructs related to body dissatisfaction, including eating disorder behaviours, are on the rise among males and adults of varying ages. ${ }^{5}$

It is necessary to understand the extent to which body dissatisfaction impairs quality of life (QoL) in order to determine the size of the public health problem that it represents. To date, relatively little research has investigated the relationship between body dissatisfaction and QoL using large population samples. In a study of 5,255 Australian women, Mond and colleagues ${ }^{6}$ found that greater body dissatisfaction was associated with poorer physical and mental health-related QoL and poorer psychosocial functioning. Further, these associations were independent of the well-documented relationship between body dissatisfaction and eating disorder symptoms. The association between body dissatisfaction and physical health-related QoL was weak in comparison to the association with mental health-related QoL, suggesting that the correlates of body dissatisfaction may be mostly psychological rather than physical.

\begin{abstract}
Background: Body dissatisfaction is associated with impairment in women's quality of life (QoL). To date, research has not examined the relationship between body dissatisfaction and men's QoL, or sex differences in this relationship.

Methods: A community sample of 966 males and 1,031 females living in Australia provided information about their body dissatisfaction, mental health and physical health-related QoL, and eating disorder symptoms. Data were analysed using three hierarchical multiple regressions and interactions between body dissatisfaction and sex were examined.

Results: For both sexes, increasing levels of body dissatisfaction were associated with poorer mental and physical health-related QoL and greater psychological distress. The adverse associations between body dissatisfaction and mental health-related QoL, and between body dissatisfaction and psychological distress, were more pronounced for males.

Conclusion: High levels of body dissatisfaction may threaten the psychological and physical wellbeing of both men and women. Body dissatisfaction appears to be a public health problem, distinct from the eating disorders and other adverse psychological phenomena for which body dissatisfaction is commonly discussed as a risk factor. Males, historically understudied and underrepresented in body image research, warrant increased empirical attention.
\end{abstract}

Key words: body image, quality of life, gender, sex differences

To date, no research has investigated the relationship between body dissatisfaction and QoL among males, or how this may differ from that for females. Evidence to suggest that body dissatisfaction is increasing among males highlights the need to address this gap in the literature. Data from two crosssectional general population surveys of about 3,000 adults living in Australia, collected in 1998 and again in 2008, showed that the prevalence of certain eating disorder behaviours, including extreme dieting and purging, increased more rapidly among males than females during this time period. ${ }^{5}$ Further, in the 2008 survey, binge eating - an eating disorder behaviour - was more strongly associated with impairments in mental health-related QoL in males than in females. ${ }^{5}$ Indirect evidence also alludes to increases in body dissatisfaction among males. Data suggests that the use of anabolic steroids, which is strongly linked to male body dissatisfaction ${ }^{7}$ as well as eating disorders and muscle dysmorphia, ${ }^{8}$ is increasing. The proportion of needle-exchange service users who reported that the last substance they injected was steroids increased from $2 \%$ to 7\% in Australia from 2007 to $2012 .{ }^{9}$ Current trends suggest that body image- and eatingrelated problems among males living in Australia are likely to grow. ${ }^{10}$

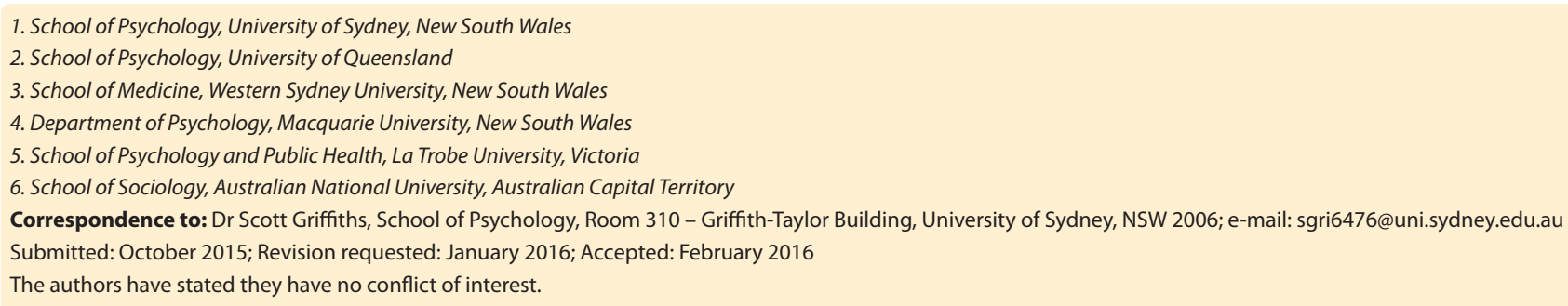


In light of the recent attention on body dissatisfaction as a public health problem and the evidence for increasing incidence of body dissatisfaction among males, this study aimed to further the emerging field of sex differences in the correlates of body dissatisfaction. To this end, we aimed to examine sex differences in the relationships between body dissatisfaction and physicaland mental health-related QoL, and between body dissatisfaction and psychological distress. We hypothesised that greater levels of body dissatisfaction would be associated with poorer health-related QoL and higher psychological distress, and that these associations would be independent of eating disorder symptoms. Given the lack of evidence pertaining to sex differences in these associations, no a priori hypotheses were made regarding interactions between sex and body dissatisfaction.

\section{Method \\ Participants}

Participants were recruited from the state of Victoria, Australia, subsequent to random selection from the Electoral Roll. In Australia, voting is compulsory for adult citizens aged 18 years and over, so the Electoral Roll provides a relatively complete database of Australian adults. A total of 9,357 adults were mailed an invitation to participate in the study; 2,099 responded and provided data, yielding a response rate of $22.4 \%$. Detailed information about the recruitment process has been published elsewhere. ${ }^{11}$

Following exclusion of participants with missing data on any of the measures assessed, the total sample of participants was 966 males and 1,031 females. On average, participants were 44 years old (range 18-67 years); slightly overweight (BMI: $\mathrm{M}=26.5$ $\left.\mathrm{kg} / \mathrm{m}^{2}, S D=5.2 \mathrm{~kg} / \mathrm{m}^{2}\right)$; married or living in a de facto relationship (70.2\%); a parent to one or more children (69.9\%); employed (78.6\%); and born in Australia (80.2\%). A minority of participants spoke a language other than English (17.4\%). Most participants were recruited from metropolitan regions $(67.4 \%)$. The median income bracket was $\$ 65,000$ to $\$ 80,000$. The sample differed from the Victorian population in that younger and middle-aged individual were underrepresented, while older individuals and individuals living in regional areas were overrepresented. ${ }^{11}$

\section{Measures}

Demographic information

Data pertaining to participants' age, sex, height and weight, marital status, employment status, country of birth, ethnicity, highest level of education, languages spoken and income bracket were collected.

\section{Body dissatisfaction}

Body dissatisfaction was measured using two items of the Eating Disorders Examination

- Questionnaire (EDE-Q), ${ }^{12}$ specifically: "How dissatisfied have you felt about your weight?" and "How dissatisfied have you felt about your shape?" Responses to each item could range from "not at all" (0) to "markedly dissatisfied" (6). Responses on these two items were highly correlated (for males, $r=0.81$, and for females, $r=0.89$ ). Thus, the responses for each item were averaged to create a single variable representing body dissatisfaction. This approach to measuring body dissatisfaction has been employed in previous studies that have examined body dissatisfaction using population samples. ${ }^{6}$

\section{Eating disorder symptoms}

The EDE-Q was used to measure eating disorder symptoms. The questionnaire assesses the occurrence and frequency of eating disorder pathology over the past month. The EDE-Q yields four sub-scales: Dietary Restraint, Eating Concerns, Weight Concerns, and Shape Concerns. Typically, a global score is computed as the average of these four subscales. To avoid statistical issues associated with multicollinearity, the two items that were used to create the aforementioned body dissatisfaction variable were omitted from the calculation of the global score. EDE-Q norms for Australian women have been reported previously and the four subscales have good reliability (a-coefficients $\geq 0.8$ ) and moderate predictive validity (sensitivity $=0.8$, specificity $=0.8$ ) in identifying probable eating disorder cases. ${ }^{13}$ Cronbach's $a$ in the present study for the global score was 0.86 for males and 0.89 for females.

\section{Health-related Quality of Life}

Mental and physical health-related QoL were measured using the Medical Outcomes Study 12-Item Short Form, Version 2 (SF12). ${ }^{14}$ The SF-12 is a standardised measure of health-related QoL. It has been used extensively in research interested in the impairment associated with physiological and psychological health conditions. Good psychometric properties have been demonstrated, including in an Australian population sample. ${ }^{14,15}$ The questionnaire has 12 items that contribute to two weighted scales, a Mental Component Summary Scale (MCS), and a Physical Component Summary Scale (PCS). Example MCS items include: "During the past four weeks, how much of the time have you felt calm and peaceful?" and "During the past four weeks, have you accomplished less than you would like as a result of any emotional problems?" Example PCS items include: "During the past four weeks, were you limited in the kind of work or other activities undertaken as a result of your physical health?" and "Does your health now limit you in moderate activities, such as moving a table, vacuuming or playing golf?" Item response scales for the MCS and PCS vary; some items are dichotomous while others offer three, five or six response options. Scores on the MCS and PCS are scaled to have a mean of 50 and standard deviation of 10, wherein higher scores indicate higher mental and physical health-related QoL, respectively. Cronbach's a scores in the present study were 0.78 for the PCS and 0.81 for the MCS.

\section{Psychological distress}

The Kessler Psychological Distress Scale $(\mathrm{K}-10)^{16}$ was used to measure psychological distress and has been developed specifically for general population samples. The K-10 includes 10 items that assess the frequency with which various depressive and anxiety symptoms are experienced. Example items include: "During the last 30 days, about how often did you feel nervous?" and "During the last 30 days, about how often did you feel worthless?"The item response scale ranges from "none of the time" (1) to "all of the time" (5). Item scores are summed such that total scores range from 10 (no distress) to 50 (maximum distress) with higher scores indicating greater levels of psychological distress. Studies suggest that scores under 20 indicate no mental disorder; scores between 20 and 24 indicate a mild mental disorder; scores between 25 and 29 indicate a moderate mental disorder; and scores 30 and above indicate a severe mental disorder. ${ }^{16,17}$ The $\mathrm{K}-10$ has been validated for use in samples of the general Australian population. ${ }^{17}$ Cronbach's a for the K-10 total score in the present study was 0.91 .

\section{Data analysis}

First, we conducted Spearman rank-order correlations to examine the unadjusted influence of body dissatisfaction on MCS 
scores, PCS scores and K10 scores. Then we conducted three hierarchical multiple regressions using MCS scores, PCS scores and $\mathrm{K} 10$ scores as the outcome variables, respectively. Each regression included two steps. At step one, the predictors included body dissatisfaction (mean-centred), eating disorder symptoms and an array of demographic variables, including sex (Table 1). The interaction term, created by calculating the product of body dissatisfaction and sex, was entered at step two.

\section{Results}

\section{Participant characteristics}

The mean level of body dissatisfaction for the full sample was $2.11(S D=1.93)$; and for males and females, mean body dissatisfaction was $1.61(S D=1.72)$ and 2.57 $(S D=1.99)$ respectively. Some level of body dissatisfaction, operationalised as a score on the body dissatisfaction variable of one or above, was reported by $70.6 \%$ of the full sample, and by $60.4 \%$ of males and $80.0 \%$ of females. Moderate to marked body dissatisfaction (score of four or above) was reported by $24.4 \%$ of the full sample and by $15.2 \%$ of males and $33.0 \%$ of females.

The mean score on the MCS for the full sample was $49.82(S D=9.70)$; and for males and females, $50.33(S D=9.37)$ and 49.35 $(S D=9.98)$, respectively. The mean score on the PCS for the full sample was 51.51 $(S D=8.87)$, and $51.13(S D=8.24)$ and 50.95 $(S D=9.37)$ for males and females, respectively. These values are comparable with normative data for the Australian population, which provides a mean MCS figure of 51.0 and a mean PCS figure of 50.5. ${ }^{18}$

The mean score on the K-10 for the full sample was $15.56(S D=5.92)$; and $15.38(S D=5.83)$ and $15.72(S D=6.01)$ for males and females, respectively. These values are comparable with normative data for the Australian population, which provides mean $\mathrm{K}-10$ figures of 14.0 for males and 15.0 for females. ${ }^{17}$

The mean global EDE-Q score for the full sample was $1.20(S D=1.10)$; and $0.99(S D=0.98)$ and $1.50(S D=1.20)$ for males and females, respectively. These values are comparable with normative data on adult women living in Australia, which provides a mean global EDE-Q score of $1.52 .{ }^{13}$ Normative data for adult men in Australia is unavailable, although the mean score obtained for males in the present study was comparable with normative data on undergraduate men enrolled at universities in the US, which provides a mean global EDE-Q score of $1.09 .{ }^{19}$ Based on published normative clinical data for eating disorders, which provides a mean global EDE-Q score of $4.06,{ }^{20}$ we determined that $0.9 \%$ of males and $3.0 \%$ of females in the present study were at risk of having an eating disorder.

\section{Correlations}

For the full sample, body dissatisfaction was significantly correlated with MCS scores, $(r=-$ $0.27, p<0.001)$, PCS scores $(r=-0.14, p<0.001)$, and K-10 scores $(r=0.34, p<0.001)$. For males only, body dissatisfaction was correlated $-0.37,-0.14$ and 0.38 , with MCS scores, PCS scores and K-10 scores, respectively $(p<0.001$ for all). For females only, body dissatisfaction was correlated $-0.24,-0.11$ and 0.31 with MCS scores, PCS scores, and K-10 scores, respectively ( $p<0.001$ for all). These relationships indicate that higher levels of body dissatisfaction are associated with lower levels of mental and physical healthrelated quality of life and with higher levels of psychological distress.

\section{Regression predicting mental health- related QoL}

The predictors entered at step one of the regression model accounted for $16.1 \%$ of the variance in MCS scores, which was significant $(\Delta F[11,1746]=30.41, p<0.001)$. The addition of the body dissatisfaction-by-sex interaction term at step two increased the proportion of variance accounted for in MCS scores by $1.1 \%$, which was also significant $(\Delta F[1$, $1745]=22.50, p<0.001)$. Therefore, individual predictors were evaluated at step two (Table 1). Eating disorder symptoms and body dissatisfaction were uniquely negatively associated with mental health-related QoL. Income, BMI and age were uniquely positively associated with mental health-related QoL. Interpreting a graphical representation of the significant interaction between sex and body dissatisfaction revealed that the association between body dissatisfaction and lower mental-health QoL was more pronounced for males compared to females (Figure 1).

\section{Regression predicting physical health- related QoL}

The predictors at step one of the regression model accounted for $17.7 \%$ of the variance in PCS scores, which was significant $(\triangle F[11$, $1746]=34.12, p<0.001)$. The addition of the body dissatisfaction-by-sex interaction term at step two increased the proportion of variance accounted for in PCS scores by less than $0.01 \%$, which was not significant $(\Delta F[1$, $1745]=0.70, p=0.402$ ). Therefore, individual predictors were evaluated at step one (Table 1). BMI, age and body dissatisfaction were uniquely negatively associated with physical health-related QoL. Income was uniquely positively associated with physical healthrelated QoL. Married and cohabitating

\begin{tabular}{|c|c|c|c|c|c|c|c|c|c|c|c|c|}
\hline \multirow{3}{*}{ Predictor } & \multicolumn{12}{|c|}{ Outcome variable } \\
\hline & \multicolumn{4}{|c|}{ Mental health-related Quality of Life } & \multicolumn{4}{|c|}{ Physical health-related Ouality of life } & \multicolumn{4}{|c|}{ Psychological distress } \\
\hline & $\beta$ & $t(1978)$ & $p$ & $\eta^{2}$ & $\beta$ & $t(1979)$ & $p$ & $\eta_{0}^{2}$ & $\beta$ & $t(1978)$ & $p$ & $\eta_{0}^{2}$ \\
\hline Body mass index & 0.11 & 4.32 & $<0.001$ & 0.011 & -0.17 & -6.61 & $<0.001$ & 0.024 & -0.14 & -5.49 & $<0.001$ & 0.017 \\
\hline Age & 0.10 & 3.52 & $<0.001$ & 0.007 & -0.19 & -6.82 & $<0.001$ & 0.026 & -0.04 & -1.49 & 0.137 & 0.001 \\
\hline Children (No $\left.{ }^{\mathrm{REF}} / \mathrm{Yes}\right)$ & 0.01 & 0.45 & 0.656 & $<0.001$ & -0.01 & -0.15 & 0.879 & $<0.001$ & -0.03 & -0.96 & 0.337 & 0.001 \\
\hline Language other than English ( $\mathrm{No}{ }^{\mathrm{REF}} / \mathrm{Yes}$ ) & -0.04 & -1.41 & 0.158 & 0.001 & -0.05 & -1.79 & 0.073 & 0.002 & 0.02 & 0.60 & 0.547 & $<0.001$ \\
\hline $\begin{array}{l}\text { Relationship status (Single, widowed or divorced } \\
\text { or cohabitating) }\end{array}$ & 0.05 & 1.63 & 0.103 & 0.002 & -0.06 & -2.05 & 0.041 & 0.002 & -0.06 & -1.96 & 0.050 & 0.002 \\
\hline Country of birth (Australia ${ }^{\mathrm{REF}} / 0$ ther) & -0.02 & -0.67 & 0.506 & $<0.001$ & -0.01 & -0.35 & 0.730 & $<0.001$ & 0.02 & 0.64 & 0.523 & $<0.001$ \\
\hline Income & 0.12 & 5.03 & $<0.001$ & 0.014 & 0.26 & 10.53 & $<0.001$ & 0.060 & -0.19 & -8.17 & $<0.001$ & 0.037 \\
\hline Region (Metropolitan ${ }^{\mathrm{REF}} /$ Regional) & 0.01 & 0.60 & 0.548 & $<0.001$ & 0.01 & 0.53 & 0.596 & $<0.001$ & -0.01 & -0.19 & 0.849 & $<0.001$ \\
\hline Eating disorder symptoms & -0.15 & -4.34 & $<0.001$ & 0.011 & 0.03 & 0.97 & 0.335 & 0.001 & 0.19 & 5.55 & $<0.001$ & 0.017 \\
\hline Body dissatisfaction & -0.36 & -8.26 & $<0.001$ & 0.038 & -0.08 & -2.27 & 0.023 & 0.003 & 0.36 & 8.26 & $<0.001$ & 0.038 \\
\hline Sex (Male ${ }^{\mathrm{REF}} /$ Female & 0.04 & 1.74 & 0.082 & 0.002 & -0.02 & -0.91 & 0.365 & $<0.001$ & -0.09 & -3.87 & $<0.001$ & 0.009 \\
\hline Interaction between sex and body dissatisfaction & 0.16 & 4.74 & $<0.001$ & 0.013 & & & & & -0.13 & -3.98 & $<0.001$ & 0.009 \\
\hline
\end{tabular}

${ }_{\mathrm{REF}}=$ Reference group

Partial eta-squared $\left(\eta_{p}^{2}\right)$ values of approximately $.01, .06$, and . 14 , indicate, small, medium, and large effect sizes, respectively. ${ }^{21}$

Summary statistics for the regressions predicting mental health-related quality of life and psychological distress were obtained from stage two of the respective hierarchical regressions. Summary statistics for the regression predicting physical health-related quality of life were obtained from stage one. 
participants reported significantly lower physical health-related QoL than single, widowed and divorced participants. Interpreting a graphical representation of the non-significant interaction between sex and body dissatisfaction (Figure 1) supported the statistical conclusion that the association between body dissatisfaction and lower physical health-related QoL was not moderated by sex.

\section{Regression predicting psychological distress}

The predictors at step one of the regression model accounted for $20.2 \%$ of the variance in $\mathrm{K} 10$ scores, which was significant $(\Delta F[11$, $1746]=40.07, p<0.001)$. The addition of the body dissatisfaction-by-sex interaction term at step two increased the proportion of variance accounted for in $\mathrm{K} 10$ scores by $0.7 \%$, which was also significant $(\Delta F[1,1745]$ $=15.83, p<0.001)$. Therefore, individual predictors were evaluated at step two (Table 1). Body dissatisfaction and eating disorder symptoms were uniquely positively associated with psychological distress. Income and BMI were uniquely negatively associated with psychological distress. Female participants reported significantly less psychological distress than male participants. Interpreting a graphical representation of the interaction between sex and body dissatisfaction (Figure 2) revealed that the association between body dissatisfaction and higher psychological distress was more pronounced for males compared to females.

\section{Discussion}

In light of the lack of research into QoL of males with body dissatisfaction, we aimed to examine sex differences in the relationships between body dissatisfaction and mental and physical wellbeing. Our hypotheses were supported, insofar as higher levels of body dissatisfaction were uniquely associated with lower mental health-related QoL, lower physical health-related QoL and greater psychological distress; controlling for BMI, eating disorder symptoms and a broad range of demographic covariates. In addition, we observed two significant interactions between body dissatisfaction and sex. Specifically, the adverse relationships between body dissatisfaction and mental health-related QoL, and between body dissatisfaction and psychological distress, were more pronounced (i.e. steeper) for men compared to women. No interaction was observed for physical health-related QoL. Thus, it appears that males with high levels of body dissatisfaction may experience, to a limited degree, greater impairment in their psychological wellbeing than females. We stress, however, that these sex differences were small in terms of effect size.

Our results showing poorer mental healthrelated QoL and greater psychological distress for males compared to females at higher levels of body dissatisfaction may be attributable to the differential levels of stigma associated with body dissatisfaction for males and females. Males experiencing high levels of body dissatisfaction may be subject to greater stigmatisation, or experience more self-stigma than females with similar levels of body dissatisfaction, as research has demonstrated the presence of stigma and self-stigma among males with eating disorders, ${ }^{22-25}$ and stigma surrounding body dissatisfaction and body image among high school boys. ${ }^{26}$ The experience of stigma may contribute to the manifestation of poorer QoL among males than females at comparable levels of body dissatisfaction. To this end, research with individuals with
Figure 1: Predicted Mental

Component Summary (MCS) for males and females

as a function of body

dissatisfaction ( $0=$ no body

dissatisfaction, $6=$ marked body dissatisfaction).

Lower MCS scores indicate poorer mental health-related quality of life. A significant interaction between body dissatisfaction and sex was observed.

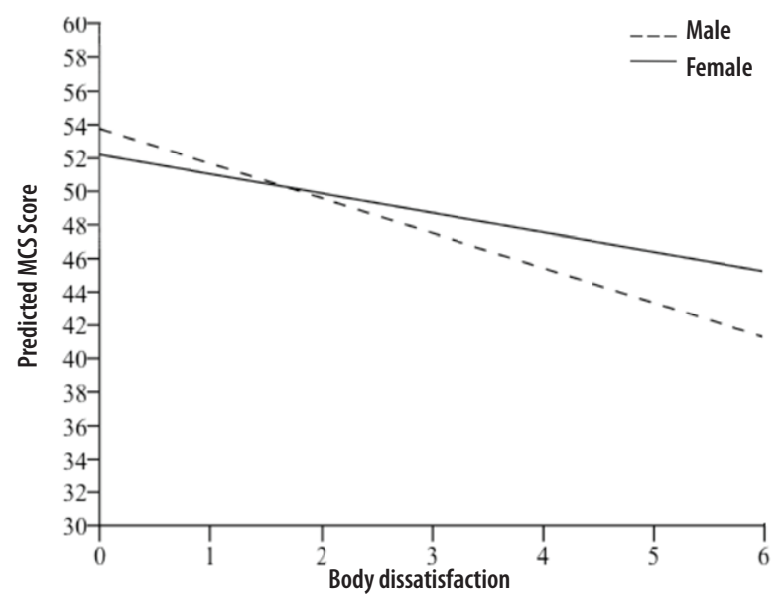

eating disorders suggests that internalisation of mental illness stigma is associated with poorer psychosocial and psychiatric outcomes. ${ }^{27}$

Alternative explanations include that males with high levels of body dissatisfaction may experience disproportionately more dissatisfaction with their muscularity relative to females, and muscularity- and thinness-dissatisfaction may differ in the strength of their relationships with QoL. Research on male body image has found different pathways through which body fat and muscle dissatisfaction influence body change behaviours, ${ }^{28}$ and an emphasis on muscle rather than body fat, and vice versa, may predispose males toward the development of muscle dysmorphia and anorexia nervosa, respectively. ${ }^{29,30}$ Another possibility is that because body dissatisfaction is less common among males compared to females, the occurrence of substantial body dissatisfaction in a male may be more likely to be indicative of more general and severe psychological problems than in females. To this end, there is some evidence to suggest that males with eating disorders may exhibit greater co-morbidity than females with eating disorders, ${ }^{31}$ including higher rates of compulsive exercise $\mathrm{s}^{32}$ and anxiety disorders, although females may be more likely to suffer from co-morbid substance use disorders. ${ }^{33}$

The results of the current study corroborate statements by researchers that body dissatisfaction is a distinct public health problem that is separate from the eating disorders and other adverse psychological sequelae for which body dissatisfaction is commonly discussed as a risk factor. ${ }^{12,34}$ In a large population sample of women living in Australia, Mond and colleagues (2012) ${ }^{6}$ showed that increasing body dissatisfaction was associated with impairment in mental and physical health-related QoL. We extended these findings by showing that the associations between body dissatisfaction and impaired mental and physical healthrelated QoL are also present for men living in Australia, and that sex moderates the relationship between body dissatisfaction and mental health-related QoL.

Implications of the present study include a re-emphasis of the need for interventions to address body dissatisfaction. To this end, researchers have made considerable progress. ${ }^{35,36} \mathrm{Few}$ body image interventions, however, have targeted males, and although our data suggest that, overall, 
the burden of body dissatisfaction is borne disproportionately by females, it appears that males with body dissatisfaction may be a particularly high-risk group. Given the trends for male eating disorder behaviours and anabolic steroid use, the results of our study underscore the need for additional research into interventions to address male body dissatisfaction.

Limitations of this study are noted. First, the male and female experiences of body dissatisfaction are distinct in that males are typically much more concerned over their level of muscularity than females. ${ }^{37}$ Arguably, the two EDE-Q items used to create the composite body dissatisfaction measure may not capture males' muscularity concerns, and body fat and muscularity dissatisfaction may have differential relationships with QoL among males. ${ }^{38}$ Second, the data were crosssectional, tempering any conclusions about causality. Third, the generalisability of the findings is uncertain, given the low response rate and the likelihood that people with a pre-existing interest in body image, eating disorders or mental health may have been more likely to respond to the study invitation. Strengths of the study are also noted, specifically, our use of a large communitybased sample that included a large number of male participants (the exclusion of whom remains common in body image research) and the novelty of our findings.

In conclusion, researchers have cautioned that the fact that dissatisfaction with weight or shape is 'normative' in industrialised nations should not be taken to infer that it is benign. ${ }^{6,34}$ Our results support this assertion and extend the findings to include males, for whom body dissatisfaction appears to be a growing public health problem and for whom the experience of body dissatisfaction may be an equally strong threat to psychological wellbeing.

\section{References}

1. Mond J, van den Berg P, Boutelle K, Hannan P, NeumarkSztainer D. Obesity, body dissatisfaction, and emotional well-being in early and late adolescence: Findings from the Project EAT study. J Adolesc Health. 2011;48:373-8.

2. Paxton S. Body image dissatisfaction, extreme weight loss behaviours: Suitable targets for public health concerns? Health Promot J Austr. 2000;10:15.

3. Stice E, Shaw HE. Role of body dissatisfaction in the onset and maintenance of eating pathology: A synthesis of research findings. I Psychosom Res. 2002;53:985-93.

4. Paxton SJ, Neumark-Sztainer D, Hannan PJ, Eisenberg ME. Body dissatisfaction prospectively predicts depressive mood and low self-esteem in adolescent girls and boys. J Clin Child Adolesc Psychol. 2006;35: 539-49.
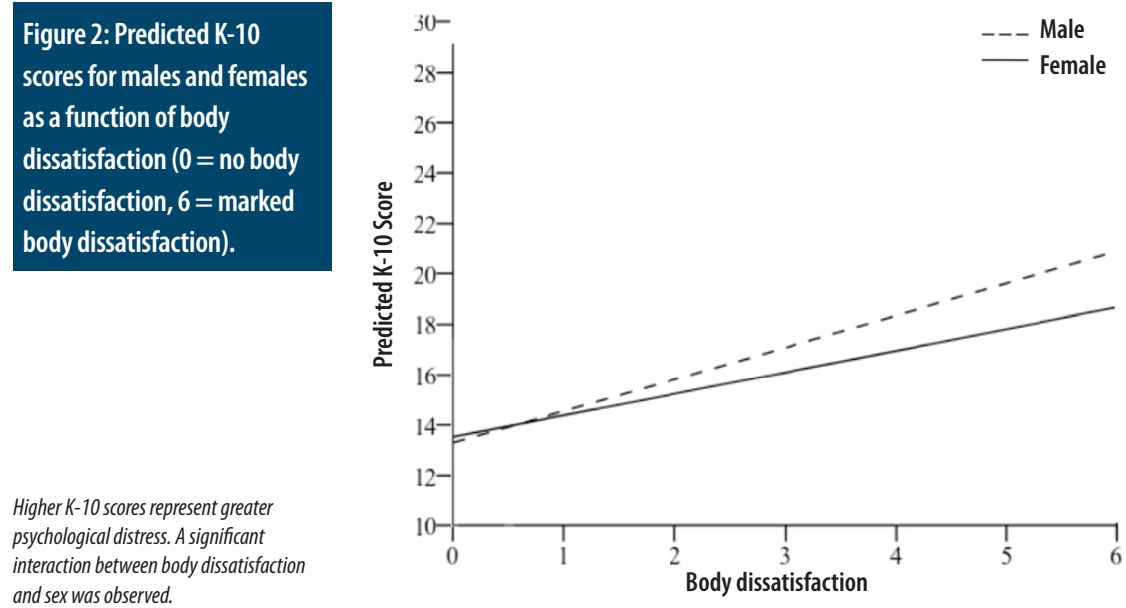

Higher K-10 scores represent greater psychological distress. A significant interaction between body dissatisfaction and sex was observed.

5. Mitchison D, Hay P, Slewa-Younan S, Mond J. The changing demographic profile of eating disorder behaviors in the community. BMC Public Health. 2014;14:943.

6. Mond J, Mitchison D, Latner J, Hay P, Owen C, Rodgers B. Quality of life impairment associated with body dissatisfaction in a general population sample of women. BMC Public Health. 2012;13:920.

7. Pope HG, Kanayama G, Hudson JI. Risk factors for illicit anabolic-androgenic steroid use in male weightlifters: A cross-sectional cohort study. Biol Psychiatry. 2012;71:254-61.

8. Olivardia R, Pope $\mathrm{H}$, Hudson J. Muscle dysmorphia in male weightlifters: A case-control study. Am JPsychiatry. 2000;157:1291-6.

9. Iversen J, Maher L. Australian Needle and Syringe Program National Data Report 2008-2012. Sydney (AUST): University of New South Wales Kirby Institute; 2013.

10. Griffiths S, Murray SB, Touyz S. Keeping pace with the growing problems of male eating disorders. Med Today. 2015;16:63-5.

11. McLean SA, Paxton SJ, Massey R, Hay PJ, Mond JM, Rodgers $B$. Stigmatizing attitudes and beliefs about bulimia nervosa: Gender, age, education and income variability in a community sample. Int J Eat Disord. 2014;47:353-61.

12. Fairburn C, Beglin S. Assessment of eating disorders: Interview or self-report questionnaire? Int J Eat Disord. 1994;16:363-70.

13. Mond JM, Hay PJ, Rodgers B, Owen C. Eating Disorder Examination Questionnaire (EDE-Q): Norms for young adult women. Behav Res Ther. 2006;44:53-62.

14. Ware J, Kosinski M, Keller SD. A 12-Item Short-Form Health Survey: Construction of scales and preliminary tests of reliability and validity. Med Care. 2002;34: 220-33.

15. Sanderson K, Andrews G. The SF-12 in the Australian population: Cross-validation of item selection. Aust $N$ ZJ Public Health. 2002;26:343-5.

16. Kessler RC, Andrews G, Colpe L, Hiripi E, Mroczek DK, Normand SLT, et al. Short screening scales to monitor population prevalences and trends in non-specific psychological distress. Psychol Med. 2002;32:959-76.

17. Slade T, Grove R, Burgess P. Kessler Psychological Distress Scale: Normative data from the 2007 Australian National Survey of Mental Health and Wellbeing. Aust NZJ Psychiatry. 2011;45:308-16.

18. TuckerG, Adams R, Wilson D. New Australian population scoring coefficients for the old version of the SF-36 and SF-12 health status questionnaires. Qual Life Res. 2010;19(7):1069-76.

19. Lavender JM, De Young KP, Anderson DA. Eating Disorder Examination Questionnaire (EDE-O): Norms for undergraduate men. Eating Behav. 2010;11:119-21.

20. Welch $E$, Birgegård A, Parling T, Ghaderi A. Eating disorder examination questionnaire and clinical impairment assessment questionnaire: General population and clinical norms for young adult women in Sweden. Behav Res Ther. 2011;49:85-91.
21. Richardson J. Eta squared and partial eta squared as measures of effect size in educational research. Educ Res Rev. 2011;6(2):135-47.

22. Griffiths S, Mond JM, Murray SB, Touyz S. The prevalence and adverse associations of stigmatization in people with eating disorders. Int J Eat Disord. 2015;48:767-74.

23. Griffiths S, Mond JM, Li Z, Gunatilake S, Murray SB, Sheffield J, et al. Self-stigma of seeking treatment and being male predict an increased likelihood of having an undiagnosed eating disorder. Int J Eat Disord. 2015;48775-8.

24. Räisänen $U$, Hunt $K$. The role of gendered constructions of eating disorders in delayed help-seeking in men: a qualitative interview study. BMJOpen. 2014;4:e004342.

25. Robinson KJ, Mountford VA, Sperlinger DJ. Being men with eating disorders: Perspectives of male eating disorder service-users.J Health Psychol. 2013;18:176-86.

26. Hargreaves DA, Tiggemann M.'Body image is for girls': A qualitative study of boys'body Image. J Health Psychol. 2006;11:567-76.

27. Griffiths S, Mond JM, Murray SB, Thornton C, Touyz S Stigma resistance in eating disorders. Soc Psychiatry Psychiatr Epidemiol. 2014;50:279-87.

28. Tylka TL. Refinement of the tripartite influence model for men: Dual body image pathways to body change behaviors. Body Image. 2011;8:199-207.

29. Griffiths S, Murray SB, Touyz S. Extending the masculinity hypothesis: An investigation of gender role conformity, body dissatisfaction, and disordered eating in young heterosexual men. PsycholMen Masc. 2015;16:108-14.

30. Murray SB, Rieger E, Karlov L, Touyz SW. Masculinity and femininity in the divergence of male body image concerns. J Eat Disord. 2013;1:1-8.

31. Weltzin TE, Cornella-Carlson T, Fitzpatrick ME, Kennington $B$, Bean $P$, Jefferies $C$. Treatment issues and outcomes for males with eating disorders. Eat

32. Murray SB, Griffiths S, Rieger E, TouyzS. A comparison of compulsive exercise in male and female presentations of anorexia nervosa: What is the difference? Adv Eat Disord. 2013;2:65-70.

33. GadallaTM. Psychiatric comorbidity in eating disorders: A comparison of men and women. J Mens Health. 2008;5:209-17.

34. Bucchianeri M, Neumark-Sztainer D. Body dissatisfaction: An overlooked public health concern. J Public Ment Health. 2014;13:64-9.

35. Bird EL, Halliwell E, Diedrichs PC, Harcourt D. Happy Being Me in the UK: A controlled evaluation of a schoolbased body image intervention with pre-adolescent children. Body Image. 2013;10:326-34.

36. Tiggemann M, Slater A, Bury B, Hawkins K, Firth B. Disclaimer labels on fashion magazine advertisements: Effects on social comparison and body dissatisfaction. Body Image. 2013;10:45-53.

37. McCreary D, Sasse DK. An exploration of the drive for muscularity in adolescent boys and girls. J Am Coll Health. 2000;48:297-304.

38. Griffiths S, Murray SB, Touyz S. Disordered eating and the muscular ideal. J Eat Disord. 2013;1:15 Disord. 2012;20:444-59. 